\title{
A design technique for optimizing resonant coils and the energy transfer of inductive links.
}

\author{
HEIDARIAN, M. and BURGESS, S.J.
}

2021

(C) 2021 IEEE. Personal use of this material is permitted. Permission from IEEE must be obtained for all other uses, in any current or future media, including reprinting/republishing this material for advertising or promotional purposes, creating new collective works, for resale or redistribution to servers or lists, or reuse of any copyrighted component of this work in other works. 


\title{
A Design Technique for Optimizing Resonant Coils and the Energy Transfer of Inductive Links
}

\author{
Maryam Heidarian $^{\circledR}$, Graduate Student Member, IEEE, and Samuel J. Burgess ${ }^{\circledR}$
}

\begin{abstract}
Power transfer efficiency (PTE) is a key performance parameter in development work on resonant inductive power transfer (IPT) systems. Geometrically optimizing the transmitter ( $\mathbf{T x})$ and receiver $(\mathbf{R x})$ coil pair is a way of improving the IPT system's efficiency. In this article, a new figure-of-merit (FoM) is proposed to find an optimum coil geometry which maximizes the PTE. The employed FoM parameter, called the "strong coupling factor" $\left(P_{\text {scf }}\right)$, is defined such that its value indicates how strongly the $T x$ and $R x$ coils are linked together. Considering the IPT application and its physical size constraints, a proper selection method for identifying the numerical value of $P_{\text {scf }}$ is essential for optimal coil geometry design. This article presents an iterative algorithm to assist in the selection of the most favorable $\boldsymbol{P}_{\text {scf }}$ value which provides maximized PTE for the designed optimum coil geometry. Design examples of two nominal IPT systems at frequencies of 415 and $0.1 \mathrm{MHz}$ are used to investigate the design algorithm. Theoretical calculations show the optimum geometry designed for the IPT system operating at $415 \mathrm{MHz}$, with coupling coefficient $(K)$ of 0.2 , can achieve maximum PTE of $85.70 \%$. Measurements presented from a practical $T x / R x$ coil pair in the IPT link operating at $0.1 \mathrm{MHz}$, with $K=0.05$, show a PTE of $83.10 \%$ against a calculated PTE of $84.11 \%$ validating the design process.
\end{abstract}

Index Terms-Inductive power transfer (IPT), magnetic resonance, power transfer efficiency (PTE), wireless power transfer (WPT).

\section{INTRODUCTION}

W IRELESS power transfer (WPT) offers a cordless method of energy transmission which can reduce the weight and cost of battery-powered devices. Inductive power transfer (IPT) is a WPT method that uses magnetic coupling between a transmitter (Tx) and a receiver $(\mathrm{Rx})$ coil. It is based on the principle that a varying magnetic field produced by a primary winding will induce a magnetic field inside a secondary winding. The primary field, when interacting with the secondary winding, will set up a current flow in a load connected to the secondary: power can be transferred as can data. Magnetic flux leakage (i.e., leakage inductance) in the energy transfer link can significantly degrade the induced power in the Rx side. Adding resonators (i.e., compensation capacitors)

Manuscript received July 8, 2020; revised September 11, 2020; accepted September 19, 2020. This work was supported by the School of Engineering, Robert Gordon University, Aberdeen, U.K. This article is an expanded version from the IEEE Wireless Power Week, London, U.K., June 17-20, 2019. (Corresponding authors: Maryam Heidarian; Samuel J. Burgess.)

The authors are with the School of Engineering, Robert Gordon University, Aberdeen AB10 7GJ, U.K. (e-mail: m.heidarian@rgu.ac.uk; s.j.burgess@ rgu.ac.uk).

Digital Object Identifier 10.1109/TMTT.2020.3028969 to the inductively coupled windings can effectively cancel out the leakage reactance of the system and bring both windings to a resonance frequency $\left(f_{o}\right)$.

Resonant inductive coupling, by providing a stronger inductive interaction between the Tx and Rx coil pairs, does improve the power transfer efficiency (PTE) of these WPT systems. However, maintaining maximum PTE in inductive links has always been a challenge [1]. In a resonant IPT link the PTE is influenced by three factors which are as follows.

1) transmission medium distance (between coils) and attenuation level;

2) terminating circuitry of both $\mathrm{Tx}$ and $\mathrm{Rx}$ sides (i.e., source resistance, $R_{s}$, and load resistance, $R_{L}$, respectively);

3) coil size (e.g., diameter, length, number of turns, etc.). The medium distance and attenuation levels directly affect the magnetic flux coupling between the Tx/Rx pair (i.e., coupling coefficient, $K$ ). The terminating impedances of the Tx/Rx pair set the circuitry power losses in the primary and secondary which, in turn, indicate the system loaded quality factor ( $Q_{L}$-factor). Also, the coil geometry determines the intrinsic quality factor $\left(Q_{i}\right.$-factor) of the $\mathrm{Tx} / \mathrm{Rx}$ coil pair which, in turn, affects their power transmission/receiving ability.

Strong inductive coupling between primary and secondary coils supports maximum PTE [2]. This requires a resonant inductive link that possesses high magnetic flux coupling while both $Q_{i}$-factor and $Q_{L}$-factor are maximized. From a practical viewpoint fulfilling these requirements is not always possible. The bulk of WPT applications have a significant air gap between Tx and Rx which limits the link's magnetic flux coupling. The application's required power level also affects both the loaded and intrinsic quality factors. The resonantly coupled coils can be used to wirelessly deliver any volume of power; from few $\mathrm{mW}$ in implantable microelectronic devices (IMDs) [1], [3]-[8] to $\mathrm{kW}$ range in electric vehicle (EV) battery chargers [9]-[11]. Managing such a range of power levels requires an IPT system with specific $R_{S}$ and $R_{L}$ values, which imposes some constraints on the system's $Q_{L}$-factor. The application requirements, such as specific power level and space limitations, also influence the structural topology of the inductive resonators (i.e., $Q_{i}$-factor). Two coil topologies commonly used in WPT applications are spiral and helical inductors. The planar structure of the spiral coils makes them more suitable for space-confined applications such as IMDs [1], [4], [7], [8] and portable electronic devices [12], [13]. On the other hand, helical coils, due to 
their uniform magnetic field [14], can provide a large inductive coupling in comparison with spiral coils [6], [9], [14]-[17]. Examples of such systems include high-voltage battery charging [11], [18], [19] and WPT through-metal-walls [20]-[22]. It should be noted that selecting the coil topology is mainly influenced by the application's spatial restriction. For example, Sallan et al. [9] and Wang et al. [10], due to the EV's limited space, have utilized spiral (planar) coils in EV battery charging with high power level requirements. These limitations and requirements necessitate optimizing the physical circuit parameters of an IPT system accordingly, which can be highly challenging.

Much work has been devoted to maximizing PTE with regards to the mentioned three impacting factors (i.e., $K$, $Q_{i}$, , and $Q_{L}$-factors). This article focuses on geometric optimization of $\mathrm{Tx} / \mathrm{Rx}$ coil pairs as a means of improving inductive linkage [1], [5]-[9], [14], [15], [23]-[32], which can additionally reduce the IPT system's physical size. One of the methods for geometric optimization of inductive coils is through increasing $Q_{i}$-factor of the Tx/Rx coil pairs which has been considered by many researchers including [24]-[27]. In the proposed technique, to improve $Q_{i}$-factor, the coil's winding layout has been modified in different ways such as varying the winding's track width [24] and increasing the coil's inner radius [26]. Employing a Tx/Rx coil pair with higher $Q_{i}$-factor can improve the energy transmission of the pair, however maximizing source-to-load PTE requires configuring the coil's geometry while compensating the loading effect of $K$ and $Q_{L}$-factor as well.

To maximize the system's overall PTE through coil geometry optimization, different iterative design algorithms have been proposed over the last few decades [1], [5]-[7], [9], [14], [15], [28]-[32]. As a common approach to cancel out the loading effect of Tx/Rx terminating circuitry, Jow and Ghovanloo [1], Ibrahim and Kiani [5], Sallan et al. [9], Hwang and Jang [15], Ko et al. [29], and Donaldson and Perkins [30] have found equations for PTE based on the system parameters (e.g., $K, R_{s}, R_{L}$, etc.). Then, for the derived PTE equation, various figure-of-merit (FoM) parameters are defined to help improve energy transmission efficiency for the purpose application. For example, to optimize the geometry of printed spiral coils in a cortical visual prothesis, as listed in Table I, the coil's diameter $(D)$, conductor width $(W)$, winding's distribution over the spiral disk $(\varphi)$, and the number of turns $(N)$ for both the Tx and Rx coils have been considered as FoM parameters by Jow and Ghovanloo [1]. In these proposed techniques, in order to determine the proper numerical combination of FoMs which maximizes the PTE, the design parameters are swept one by one in a wide range around their preselected initial values. This process is repeated until the considered combination lead to a desired PTE level. The selected initial FoMs and their respective designed optimum values, for the previous example, are shown in Table I. As can be seen, the optimized FoM values have significant differences from their preliminary values which indicates the high number of taken iterations. Using this method respective measured PTEs of $41.2 \%$ at $f_{o}=1 \mathrm{MHz}$ and $85.8 \%$ at $f_{o}=5 \mathrm{MHz}$
TABLE I

FOM PARAMETERS AND DESIGN VALUES FOR THE IPT APPLICATION IN [1]

\begin{tabular}{lccc}
\hline \hline FoM parameters & Symbols & $\begin{array}{c}\text { Initial } \\
\text { values }\end{array}$ & $\begin{array}{c}\text { Optimised } \\
\text { values }\end{array}$ \\
\hline Diameter - Tx coil & $D_{T x}(\mathrm{~mm})$ & 20 & 70 \\
Diameter - Rx coil & $D_{R x}(\mathrm{~mm})$ & 8 & 20 \\
Conductor width - Tx coil & $W_{T x}(\mu \mathrm{m})$ & 38 & 3250 \\
Conductor width - Rx coil & $W_{R x}(\mu \mathrm{m})$ & 38 & 250 \\
Fill factor - Tx coil & $\varphi_{T x}$ & 0.43 & 0.85 \\
Fill factor - Rx coil & $\varphi_{R x}$ & 0.43 & 0.43 \\
Number of turns - Tx coil & $N_{T x}$ & 71 & 9 \\
Number of turns - Rx coil & $N_{R x}$ & 31 & 15 \\
\hline \hline
\end{tabular}

are found for a transmission distance of $10 \mathrm{~mm}$ and a $500-\Omega$ resistive load.

Depending on the number of geometrical variables (i.e., FoMs) that need to be swept, the proposed coil optimization techniques clearly are very time consuming. To accelerate finding the optimal FoM parameters and make the design process more intuitive, Ahn and Ghovanloo [6] and Cheng et al. [14] have defined a combination of the design parameters as the FoM maximizing the PTE. To optimize the geometry of Rx coil, Cheng et al. [14] has considered multiplication of the Rx's $Q_{L}$-factor, a part of the inductive link's coupling coefficient and the ratio of power delivery to the load as the only required FoM. Utilizing the defined FoM parameter, a four-turn solenoid using a $0.1270-\mathrm{mm}$ (dia) copper wire was prototyped for millimeter-sized IMDs operating at $700 \mathrm{MHz}$. The proposed method only focuses on optimizing Rx coil geometry to improve the PTE. Also, there is no indication of the system's required power level and achieved PTE. To consider the effect of both Tx and Rx in maximizing the system's source-to-load PTE, Ahn and Ghovanloo [6] has defined two independent FoMs (i.e., Rx-FoM and Tx-FoM) representing the roles of primary and secondary sides. The $\mathrm{Rx}$-FoM has been considered such that its value indicates how efficiently the application's load can receive the power. This is achieved through multiplication of the Rx's $Q_{L}$-factor and the ratio of power delivery to the load. The Tx-FoM is defined as the link's coupling coefficient squared multiplied by the Tx $Q_{i}$-factor (i.e., $K^{2} Q_{i_{p}}$ ). The multiplication of Rx- and Tx-FoMs provides the IPT system's overall PTE. Utilizing this technique, the first step to improve the PTE is to maximize the Rx-FoM, then the Tx coil geometry is optimized with considering the designed Rx. Using the developed Tx and Rx coils, the overall PTE of $1.02 \%$ could be measured while the power delivered to the load was $224 \mu \mathrm{W}$ at $200 \mathrm{MHz}$ with $12-\mathrm{mm}$ distance (i.e., estimated $K=0.002$ ) between the primary and secondary. In the proposed approach the inductive link's coupling coefficient is considered as a property of Tx-FoM. However, $K$ based on its definition is affected by both the Tx and Rx [14]. We, in our previous article [31], 
have modified the approach presented in [6] by considering only one FoM parameter such that its value indicates how strongly both the Tx and Rx sides are linked together. The proposed FoM parameter, named as "strong coupling factor" $\left(P_{\text {scf }}\right)$, has been defined as: $P_{\text {scf }}=K^{2} Q_{L_{p}} Q_{L_{s}}$. The theoretical method in [31] was practically exemplified by the design of an optimized $\mathrm{Tx} / \mathrm{Rx}$ solenoid pair geometry for an inductive link with a transmission distance of $9.5 \mathrm{~mm}(K=0.215)$ at $f_{o}=1 \mathrm{MHz}$ [32]: a PTE of $86 \%$ was measured thus showing merit in the design approach. However, a proper selection method for identifying a numerical value to represent the FoM parameter (i.e., $P_{\text {scf }}$ ) used in [31] and [32] still requires in-depth analysis.

Building upon the proposed coil optimization technique used in [31] and [32], this article introduces a method to assist selecting the most favorable numerical value for the strong coupling factor which can maximize PTE for the designed optimum coil geometry. The proposed method will be developed through geometrical optimization of a helical coil (i.e., solenoid). A solenoid has been chosen since the available coil design algorithms in the literature mainly focus on spiral coils or low power (i.e., $\ll 1 \mathrm{~W}$ ) $\mathrm{mm}$-size solenoids. However, geometrical optimization of high-power helical coils can be equally important. This is because for the coils to be suitable for high power IPT applications, the coil winding conductor must be large enough to tolerate the transmitting current level. This requirement yields a bulky coil geometry which in a physical implementation can encounter spatial difficulties. For example, in a typical WPT application transmitting 0.5-5-W power through-metal, a solenoid with a diameter of $84 \mathrm{~mm}$, length of $152 \mathrm{~mm}$, and 260 turns is required to operate at $0.3 \mathrm{MHz}$ [20], geometrical optimization of the Tx/Rx coil, besides improving the PTE, can significantly reduce the system's overall size. In addition to this, the proposed geometry optimization technique can also advantage low power IPT applications with sizable Tx/Rx helical coils. One example of such applications is the capsule endoscopy system presented in [33], where a 120 turns Tx solenoid operating at $f_{o}=$ $0.75 \mathrm{MHz}$, delivers $39-\mathrm{mW}$ power to the $\mathrm{Rx}$.

This article is organized as follows: in Section II the physical parameters affecting the PTE of a resonant inductive WPT system are theoretically analyzed and the FoM parameter (i.e., $\left.P_{\text {scf }}\right)$ required to maximize PTE is discussed. The effect of strong coupling factor on the physical size of coil and the system's PTE has been investigated in Section III. An iterative design algorithm for proper selection of the FoM's numerical value and the subsequent optimization of coil geometry is presented in Section IV. In Section V, functionality of the proposed technique has been demonstrated by optimizing the coil geometry for a typical IPT system, similar to the WPT application in [20] as discussed above. The practical PTE measurements of the exemplified system have been presented in Section V followed by concluding remarks in Section VI.

\section{Theoretical AnAlysis on MAXIMIZING THE PTE AND THE REQUIRED FOM}

A generic lumped circuit diagram of the resonant IPT system is shown in Fig. 1 [34]. The series-series compensation

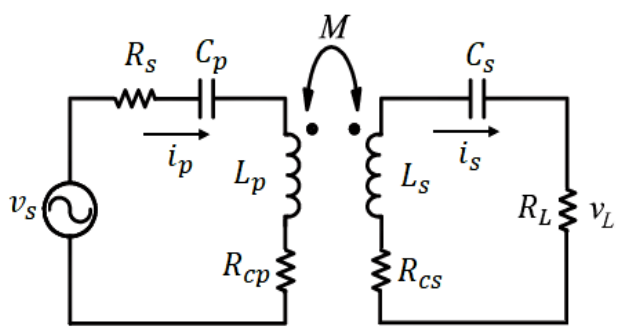

Fig. 1. Equivalent circuit model for a resonant inductive link.

topology has been considered as the connecting configuration of LC-resonators to the Tx and Rx sides. This is because this topology, at $f_{o}$, by canceling out the reflected reactance at both the primary [9] and the secondary [10] sides can provide a purely resistive inductive link. In this circuit model, $v_{s}$ and $R_{S}$ represent the ac voltage source and its equivalent internal resistance. $L_{p}$ and $L_{s}$ are the inductance values of the Tx and Rx windings, while $C_{p}$ and $C_{s}$ are the respective primary and secondary resonance compensation capacitors. $R_{\mathrm{cp}}$ and $R_{\mathrm{cs}}$ are the Tx and Rx windings' ac ohmic resistances and $M$ is the mutual inductance between Tx and Rx coil pair. Also, the equivalent ac/dc load resistance, which power is being delivered to, is indicated by $R_{L}$. Following the common approach utilized in [1], [5]-[7], the equivalent source and load resistances (i.e., $R_{s}$ and $R_{L}$ ) have been considered to represent the rest of the system's electronics. This is because in an IPT system, the application's requirements and limitations demand different types of power amplifier (e.g., classE power amplifier [35], full-bridge type inverter [11], etc.) and power management units (e.g., buck-boost converter [34], rectifier [36], dc-dc converter [37], [38], etc.) to be utilized at the primary and secondary sides, respectively. Maximizing PTE of these units have been extensively studied in the literature [35]-[38] and is out of the focus of this article [1].

At resonance, $\omega_{o}$, the application of Kirchhoff's voltage law (KVL) to the inductive link in Fig. 1 can be expressed as

$$
\left[\begin{array}{c}
V_{s} \\
V_{L}
\end{array}\right]=\left[\begin{array}{cc}
R_{s}+R_{\mathrm{cp}} & j M \omega_{o} \\
j M \omega_{o} & -R_{\mathrm{cs}}
\end{array}\right]\left[\begin{array}{l}
\mathbf{I}_{\mathbf{p}} \\
\mathbf{I}_{\mathrm{s}}
\end{array}\right]
$$

where $V_{s}$ is the rms value of sinusoidal ac voltage source and $V_{L}$ is the voltage drop over the load. Also, $\mathbf{I}_{\mathbf{p}}$ and $\mathbf{I}_{\mathbf{s}}$ are the phasors of current in the Tx and Rx sides, respectively. In order to simplify the theoretical equations and ease duplex communication between $\mathrm{Tx} / \mathrm{Rx}$, both primary and secondary coils are considered identical (i.e., $L_{p}=L_{s}=L$ and $\left.R_{\mathrm{cp}}=R_{\mathrm{cs}}=R\right)$. Hence, the PTE $(\eta)$, with respect to (1), can be defined as

$$
\begin{aligned}
\eta & =\frac{V_{L}\left|\mathbf{I}_{\mathbf{s}}\right|}{V_{s}\left|\mathbf{I}_{\mathbf{p}}\right|} \\
& =\frac{R_{L} \omega_{o}^{2} M^{2}}{\left(R_{L}+R\right)\left[\left(R_{s}+R\right)\left(R_{L}+R\right)+\omega_{o}^{2} M^{2}\right]} .
\end{aligned}
$$

Equation (2) based on the system quality factors can be restated as

$$
\eta=\frac{K^{2} Q_{L_{p}} Q_{L_{s}}}{1+K^{2} Q_{L_{p}} Q_{L_{s}}}\left(1-\frac{Q_{L_{s}}}{Q_{i}}\right)
$$


where $K$ is the coupling coefficient between the coils, $K=$ $M /\left(L_{p} L_{s}\right)^{1 / 2}=M / L$. The coil's $Q_{i}$-factor and the Tx and Rx side's $Q_{L}$-factor are, respectively, included as

$$
\begin{aligned}
Q_{i} & =\omega_{o} L / R \\
Q_{L_{p}} & =\omega_{o} L /\left(R+R_{S}\right) \\
Q_{L_{s}} & =\omega_{o} L /\left(R+R_{L}\right) .
\end{aligned}
$$

From (3) a condition which guarantees maximum PTE (i.e., $\eta=1$ ) can be expressed as

$$
\left\{\begin{array}{l}
Q_{i}-Q_{L_{s}} \equiv Q_{i} \\
K^{2} Q_{L_{p}} Q_{L_{s}} \equiv 1+K^{2} Q_{L_{p}} Q_{L_{s}} .
\end{array}\right.
$$

These require both the following inequalities to be satisfied:

$$
\left\{\begin{array}{l}
Q_{i} \gg Q_{L_{s}} \\
K^{2} Q_{L_{p}} Q_{L_{s}} \gg 1 .
\end{array}\right.
$$

With proper coil wire selection (9) is met, i.e., a low ac ohmic resistance yields a large $Q_{i}$-factor. Satisfying (10) requires.

\section{A. Inductive Link With High Coupling Coefficient " $K$ "}

Depending on the IPT system's application, the transmission medium gap and coils' mutual orientation set $K$ between 0 and 1 (i.e., $0 \leq K \leq 1$ ).

\section{B. Transceiver System With High Primary and Secondary $Q_{L}$-Factors $\left(Q_{L_{p}}\right.$ and $\left.Q_{L_{s}}\right)$}

Based on (5), a high $Q_{L_{p}}$ value can be achieved if the Tx coil has high self-inductance and small ac ohmic resistance. Also, the $R_{S}$ value should be as small as possible given the level of power dissipated in the source internal resistance what power remains for the inductive link. Based on (6), achieving a high $Q_{L_{s}}$ value also requires a coil with high self-inductance and small ac ohmic resistance. However, there are different factors affecting an IPT system's load resistance value, such as: power level (needed for the application), the inductive link's coupling coefficient and geometric parameters of $\mathrm{Tx} / \mathrm{Rx}$ coil pair. Hence, there is an optimum $R_{L}$ value for a given IPT system to satisfy operational requirements. By differentiating $\eta$ with respect to $R_{L}$ the optimum load value which maximizes PTE can be expressed as

$$
R_{L_{O}}=R \sqrt{1+\frac{\omega_{o}^{2} K^{2} L^{2}}{R\left(R+R_{S}\right)}}
$$

\section{Tx and Rx Coils With High $Q_{i}$-Factor}

Increasing a coil's $Q_{i}$-factor basically improves its power transmitting/receiving ability, which from (4) requires a coil with high self-inductance and small ac ohmic resistance. Furthermore, a high $Q_{i}$-factor coil (in addition to improving the Tx/Rx side's $Q_{L}$-factor) can ease the task of meeting (9) to maximize PTE. Equation (12) expresses coil (i.e., coreless solenoid) self-inductance according to [39]

$$
L=\frac{\mu_{o} \pi N^{2} r_{c}^{2}}{\sqrt{4 r_{c}^{2}+l_{c}^{2}}}
$$

in which $\mu_{o}$ is free space permeability, $N$ is the number of turns, $r_{c}$ is the coil radius, and $l_{c}$ is the coil length (where $l_{c} \gg r_{c}$ ). Also, the coil ac ohmic resistance including skineffect loss, based on [2], can be calculated as

$$
R=R_{\mathrm{dc}} \frac{r_{c}}{2 \delta}
$$

In (13), skin depth is represented by $\delta=\left(\rho / \pi \mu f_{o}\right)^{1 / 2}$, in which $\mu$ and $\rho$ are, respectively, the permeability and resistivity of winding wire material. $R_{\mathrm{dc}}$, based on [1], can be expressed as

$$
R_{\mathrm{dc}}=\frac{4 \rho N}{d_{w}}
$$

where $d_{w}$ is wire diameter. With replacing $R_{\mathrm{dc}}$ in (13), coil ac ohmic resistance, based on [40] and [41], can be restated as

$$
R=\frac{N r_{c} \sqrt{2 \omega_{o} \mu \rho}}{d_{w}}
$$

From (12), increasing $N$ and $r_{c}$ clearly improves coil selfinductance. However, increasing these values also has the detrimental effect of raising the coil ac ohmic resistance.

The challenge to maximize PTE is thus to design a geometrically optimized coil with maximized $L$ and low $R$. This requires exploiting the coil self-inductance to yield an ohmic resistance that provides maximized inductance coupling between Tx and Rx coil pair. The coil self-inductance, (12), is essentially a factor of three variables; $N, r_{c}$, and $l_{c}$. In a tightly wound coil $l_{c}=N d_{w}$. Expressing $N$ from a rearranged (14) and replacing it in (12) permits $L$ to be restated, only based on $r_{c}$, as

$$
L=\frac{\mu_{o} \pi R^{2} d_{w}^{2} r_{c}}{\sqrt{\left(2 \omega_{o} \mu \rho\right)\left(R^{2} d_{w}^{4}+8 r_{c}^{4} \omega_{o} \mu \rho\right)}} .
$$

Differentiating (15) with respect to $r_{c}$ provides a coil radius which maximizes $L$

$$
r_{c}=\sqrt[4]{\frac{R^{2} d_{w}^{4}}{8 \omega_{o} \mu \rho}}=\frac{l_{c}}{2} \Rightarrow L_{\max }=\sqrt[4]{\frac{\mu_{o}^{4} \pi^{4} d_{w}^{4} R^{6}}{128\left(\omega_{o} \mu \rho\right)^{3}}} .
$$

In selecting a suitable coil ac ohmic resistance, an FoM parameter $\left(P_{\text {scf }}\right)$ has been introduced, in [31] and [32], such that its value indicates how strongly the Tx and Rx coils are linked together. The constant parameter $P_{\text {scf }}\left(P_{\text {scf }} \gg 1\right)$ has been named as strong coupling factor and provides a guideline for designing a geometrically optimized coil. With considering the FoM parameter $P_{\text {scf }}$, (10) can be expressed as

$$
K^{2} Q_{L_{p}} Q_{L_{s}}=P_{\mathrm{scf}} \Rightarrow \omega_{o}^{2} K^{2} L^{2}=P_{\mathrm{scf}}\left(R+R_{s}\right)\left(R+R_{L}\right) .
$$

As the coil self-inductance needs to be maximized, we substitute $L$ with $L_{\max }$ in (17) which yields

$$
\left(\frac{\left(K \omega_{o} \mu_{o} \pi d_{w}\right)^{2}}{4 P_{\mathrm{scf}}\left(\sqrt{2 \omega_{o} \mu \rho}\right)^{3}}\right) R^{3}-R^{2}-\left(R_{s}+R_{L}\right) R-R_{S} R_{L}=0 .
$$

Solving (18) for $R$ determines the ac ohmic resistance which maximizes $\eta$ for a given resonant IPT system. From (18) it can be deduced that the optimal $R$ value is a function of $K$. 


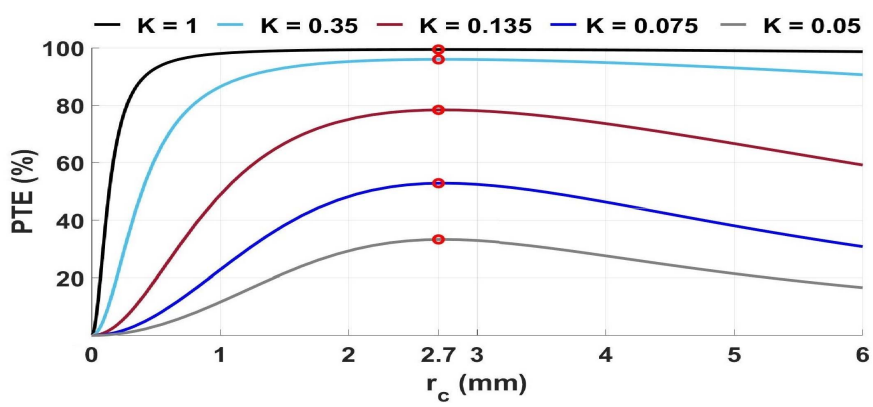

Fig. 2. Maximum PTE for different coupling coefficients $(K)$ when $P_{\text {scf }}=200$.

The inductive link's coupling coefficient is also dependent on the coil's radius $\left(r_{c}\right)$ and the relative transmission distance between Tx and Rx pair $\left(d_{t}\right)$ [16], [30]. Hence, the smallest variations in the value of coupling coefficient can easily drag the system's PTE out of the maximum $\eta$ ridge. It should be noted that based on the definition of coupling coefficient (i.e., $\left.K=M /\left(L_{p} L_{s}\right)^{1 / 2}\right)$, the coil's number of turns does not affect $K$, since both the coil's self- and mutual-inductance have the same level of dependence on $N$ [6], [14], [16], [30], [42].

To determine a unique optimum $R$ value which is robust to $K$ variations, (18) will be solved for $K=1$. This is because, as can be deduced from (17), designing a coil for the highest possible coupling coefficient can ensure the $P_{\text {scf }}$ value is large enough to maximize the PTE over the range of $0 \leq \mathrm{K} \leq 1$. Considering this, (18) can be restated as

$$
\left(\frac{\left(\omega_{o} \mu_{o} \pi d_{w}\right)^{2}}{4 P_{\mathrm{scf}}\left(\sqrt{2 \omega_{o} \mu \rho}\right)^{3}}\right) R^{3}-R^{2}-\left(R_{s}+R_{L}\right) R-R_{s} R_{L}=0
$$

The solutions to (19) can be easily found using, for instance, MATLAB. With $R$ established, $r_{c}, N$, and $l_{c}$ can be calculated from (14) and (16).

To provide an example in this regard, a typical IPT system similar to Fig. 1 , with $R_{s}=10 \Omega$ and $R_{L}=300 \Omega$ at $f_{o}=700 \mathrm{MHz}$ has been considered. For an arbitrary $P_{\text {scf }}$ value of $200\left(P_{\text {scf }}=200\right)$ the calculated ac ohmic resistance from (19) is $R=323.39 \mathrm{~m} \Omega$. For this $R$ value $r_{c}=2.70 \mathrm{~mm}$, $N=7$ and $l_{c}=5.60 \mathrm{~mm}$. For the given IPT system, the PTE variations over a range of $r_{c}$ values have been plotted in Fig. 2, with the designed geometry shown by use of red circles. It should be noted that, although the coil geometry was designed for $K=1$, it provides the maximum PTE for the range of coupling coefficient values (i.e., $0.05 \leq K \leq 1$ ). In Fig. 2, the maximum achievable PTE is seen to drop with a reduction in $K$, however the maximum PTE still stays centered around $r_{c}=2.70 \mathrm{~mm}$.

\section{Strong Coupling Factor Selection}

Achieving an optimum coil geometry, which maximizes the PTE of an IPT system, requires proper selection of numerical value for the FoM parameter (i.e., $P_{\text {scf }}$ ). Besides influencing the system's PTE the design parameter $P_{\text {scf }}$, based on (19), affects the coil ac ohmic resistance, which directly impacts the coil's physical size and $R_{L_{O}}$. To investigate the
TABLE II

Designed CoIl Geometries and PTE for Three Arbitrary CHOSEN STRONG COUPLing FACTORS

\begin{tabular}{ccccccc}
\hline \hline$P_{\text {scf }}$ & $\begin{array}{c}R \\
(m \Omega)\end{array}$ & $\begin{array}{c}r_{c} \\
(\mathrm{~mm})\end{array}$ & $N$ & $\begin{array}{c}l_{c} \\
(\mathrm{~mm})\end{array}$ & $\begin{array}{c}\eta \text { at } \\
K=0.125\end{array}$ & $\begin{array}{c}\eta \text { at } \\
K=1\end{array}$ \\
\hline 1550 & 286.64 & 6.90 & 17 & 13.80 & 95.76 & 99.65 \\
2550 & 358.17 & 7.70 & 19 & 15.50 & 97.20 & 99.60 \\
3550 & 416.24 & 8.30 & 21 & 16.70 & 97.82 & 99.56 \\
\hline \hline
\end{tabular}

strong coupling factor's effect on the physical size of Tx/Rx coil pair and the PTE, an IPT system with $R_{s}=0.1 \Omega$ and $R_{L}=100 \Omega$ at $f_{o}=13.56 \mathrm{MHz}$ has been considered. For the given system, the coil geometric parameters (i.e., $r_{c}, N$, and $l_{c}$ ) are calculated for three arbitrary chosen FoM values; $P_{\text {scf }}=1550,2550$, and 3550, as listed in Table II. Considering the derived coil geometries, the PTE variations of the designed inductive links over a range of $r_{c}$ values are plotted in Fig. 3. The IPT system's coupling coefficient values are $K=0.025,0.125$, and 1 in Fig. 3(a)-(c), respectively. The maximum achievable PTEs are marked with black circles on the curves, while their numerical values are indicated either on the graph or in Table II. From the graphs and numerical values, it can be deduced that increasing the strong coupling factor raises the coil's physical size while the maximum achievable PTE of the given IPT system is centered around the designed geometries. It should be noted that there is always a tradeoff between reducing the physical size of the coil and increasing PTE for a given $K$. Furthermore, when comparing the figures an observation is that for the inductive links with $K<1$, i.e., $K=0.025$ in Fig. 3(a) and $K=0.125$

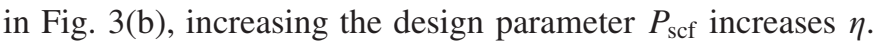
However, as $K$ gets closer to 1, as shown in Fig. 3(c), maximum PTE will slightly reduce with an increase of $P_{\text {scf }}$. This is because when $K \simeq 1$, (3) can be restated as

$$
\eta=\frac{P_{\text {scf }}}{1+P_{\text {scf }}}\left(\frac{R_{L}}{R+R_{L}}\right) .
$$

At high $P_{\text {scf }}$ values (20) is considered as

$$
\frac{P_{\text {scf }}}{1+P_{\text {scf }}} \simeq 1 \Rightarrow \eta \simeq \frac{R_{L}}{R+R_{L}}
$$

where with an increase of $P_{\text {scf }}$ the coil's ac ohmic resistance also raises leading to reduction of $\eta$.

To analyze the effect of strong coupling factor on the system's optimum $R_{L}$ value, PTE variations of the same IPT system over a range of load values are plotted in Fig. 4. The inductive link's coupling coefficient is considered as $K=0.025$ and the used $P_{\text {scf }}$ values are $P_{\text {scf }}=1550,2550$, and 3550. It can be seen that increasing strong coupling factor, due to raising $R$, increases the IPT system's optimal load value as expressed in (11). In selecting a proper $P_{\text {scf }}$ value it should be noted that achieving maximum PTE in a given inductive link requires the system's load to be equal to its optimal value from (11). However, due to many factors such as variations in coupling coefficient and charging/discharging of batteries (load), it is not always possible to keep $R_{L}$ fixed at 


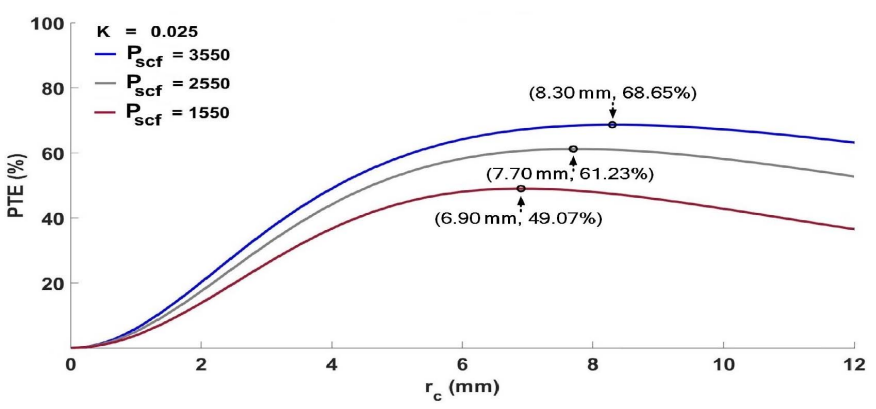

(a)

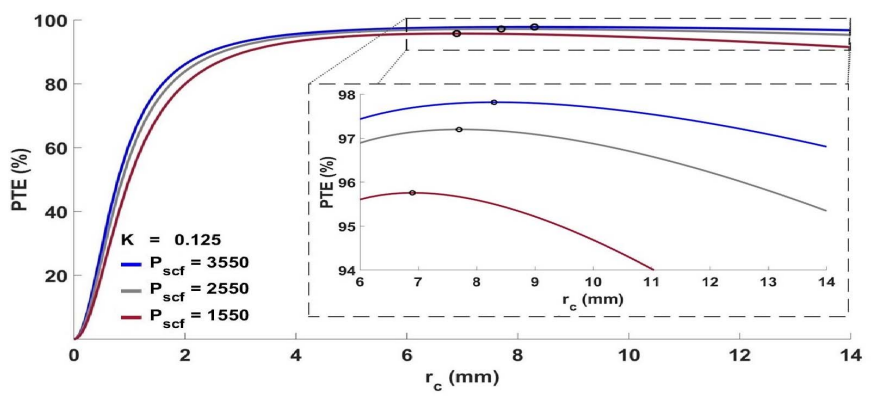

(b)

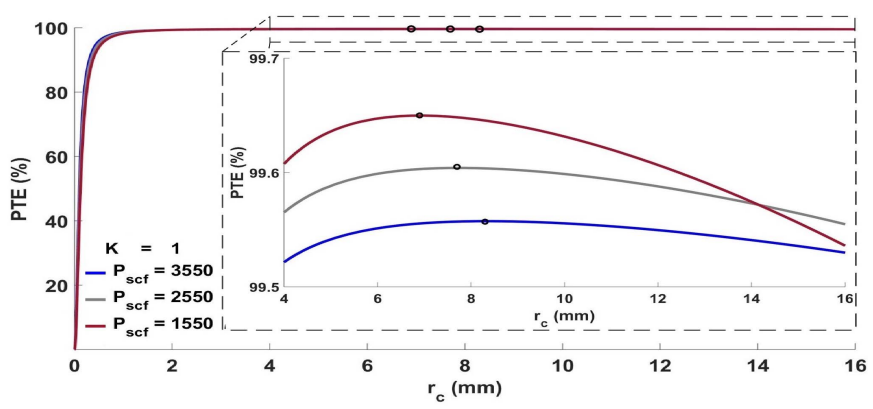

(c)

Fig. 3. Effect of increasing $P_{\text {scf }}$ on the physical size of coil and the system's PTE. Three arbitrary strong coupling factor values $\left(P_{\text {scf }}=1550\right.$, 2550, and 3550) have been chosen for (a) $K=0.025$, (b) $K=0.125$, and (c) $K=1$.

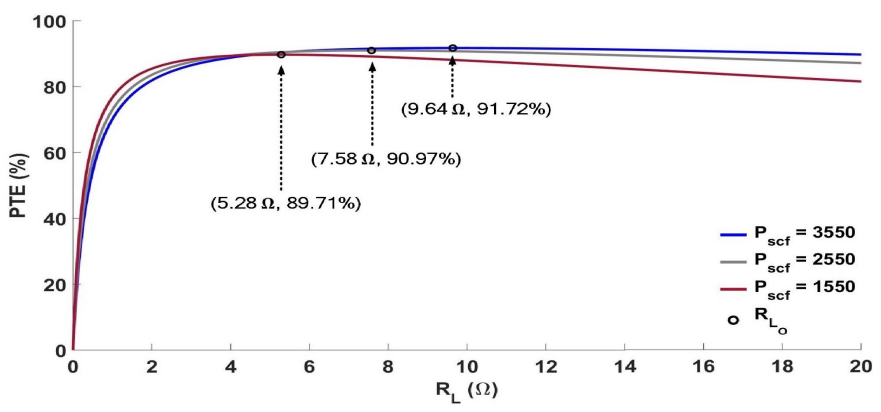

Fig. 4. Effect of increasing $P_{\text {scf }}\left(P_{\text {scf }}=1550,2550\right.$, and 3550) on the system optimal $R_{L}$ value for $K=0.025$.

the optimal load value to receive maximum PTE. To make the strong coupling factor robust to load variations a boundary $(\Delta)$ around $R_{L_{O}}$ has been introduced, where PTE of the system stays within close proximity of the maximum achievable $\eta$ (i.e., $\eta_{\max }$ ). As shown in Fig. 5, the proximity range can be any arbitrary value (e.g., $X \%$ ) dependent on the IPT system's requirement. Also, the ranges for the boundary $\Delta$ are marked

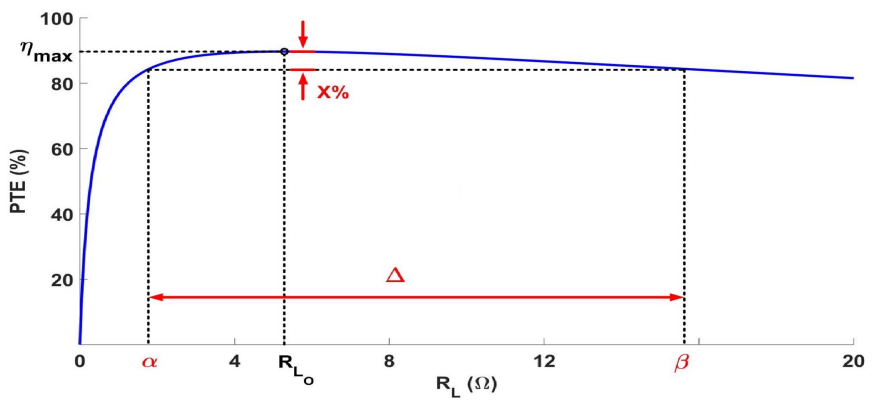

Fig. 5. Demonstration of the $\Delta$ window on the plot of PTE against load variations for the given IPT system (with $P_{\text {scf }}=1550$ and $K=0.025$ ).

by parameters $\alpha$ and $\beta$, which can be calculated from (A4) and (A5), respectively. As far as the physical size limitations permit, the most adequate $P_{\text {scf }}$ value for designing an optimum coil geometry is where the IPT system's load value sits within the $\Delta$ range (i.e., $\alpha \leq R_{L} \leq \beta$ ). To assist depicting the parameters $\alpha, \beta$, and the boundary $\Delta$, the PTE of the given IPT system for $P_{\text {scf }}=1550$ and $K=0.025$ over a range of $R_{L}$ values is shown in Fig. 5. Considering the factors impacting optimum coil geometry design (i.e., physical size, required PTE for the given IPT application and the maximum $\eta$ achievable at $R_{L_{O}}$ ), choosing a proper $P_{\text {scf }}$ value requires an iterative selection procedure.

\section{Design Procedure}

In order to find the optimal value for the FoM parameter, an iterative algorithm is depicted in Fig. 6. The demonstrated design procedure, through selecting a proper $P_{\text {scf }}$ value will provide an optimum coil geometry which maximizes PTE for the required IPT application. The algorithm starts with taking a set of design constrains and an initial value for the FoM parameter. The design constrains comprise $R_{S}$, $R_{L}, f_{o}, d_{w}, K$, the parameter $X$ and the spatial limitations (e.g., maximum $r_{c}$, etc.) which can be determined from the IPT application requirements. The FoM parameter can initially take any arbitrary value, because the optimization algorithm provides the designer with a good sense of how to make necessary changes to the $P_{\text {scf }}$ value to maximize the PTE.

To numerically describe the optimization algorithm, an arbitrary IPT system with $R_{s}=10 \Omega, R_{L}=5 \mathrm{k} \Omega$ at $f_{o}=$ $415 \mathrm{MHz}$ has been considered, where the Tx/Rx coil diameter must be less than $10 \mathrm{~mm}$ (i.e., $r_{c} \leq 5 \mathrm{~mm}$ ). The coil winding conductor used is $0.8-\mathrm{mm}$ (dia) copper wire, and the inductive link's coupling coefficient, $K$, is 0.2 . To ensure the designed coil geometry exhibits a high PTE the chosen proximity range of $\eta_{\max }$ is $10 \%$ (i.e., $X=10 \%$ ). The iterative design process is initiated with considering $P_{\text {scf }}=2000$. Table III shows some iteration examples for finding the FoM's optimal value. As can be seen in the table, to fulfill the IPT application's spatial requirements, the selected FoM values in iterations 1 and 2 have been reduced to $P_{\text {scf }}=20$ in the iteration 3. However, the coil geometry designed at the third iteration does not provide high level of PTE. Hence, to improve $\eta$, the FoM value has been slightly increased in iterations 4 and 5 . The maximum strong coupling factor value that can meet the 
TABLE III

Algorithm Iterations For IPT System Under CONSIDERATION $\left(f_{o}=415 \mathrm{MHz}, R_{s}=10 \Omega\right.$, AND $\left.R_{L}=5 \mathrm{k} \Omega\right)$

\begin{tabular}{ccccccccccc}
\hline \hline $\begin{array}{c}\text { Iteration } \\
\#\end{array}$ & $P_{\text {scf }}$ & $R$ & $r_{c}$ & $N$ & $l_{c}$ & $R_{L_{O}}$ & $\alpha$ & $\beta$ & $\eta$ for & $\eta$ for \\
$\#$ & & $(\Omega)$ & $(\mathrm{mm})$ & & $(\mathrm{mm})$ & $(\Omega)$ & $(\Omega)$ & $(\Omega)$ & $R_{L_{O}}(\%)$ & $R_{L}(\%)$ \\
\hline 1 & 2000 & 2.04 & 7.80 & 20 & 15.80 & - & - & - & - & - \\
2 & 200 & 0.92 & 5.30 & 13 & 10.40 & - & - & - & - & - \\
3 & 20 & 0.42 & 3.60 & 9 & 7.00 & 40.96 & 3.15 & 532.29 & 97.97 & 44.43 \\
4 & 100 & 0.72 & 4.70 & 11 & 9.30 & 120.36 & 5.83 & 2484.15 & 98.80 & 79.99 \\
\hline 5 & 150 & 0.83 & 5.00 & 12 & 10.00 & 157.98 & 6.78 & 3678.36 & 98.95 & 85.70 \\
\hline \hline
\end{tabular}

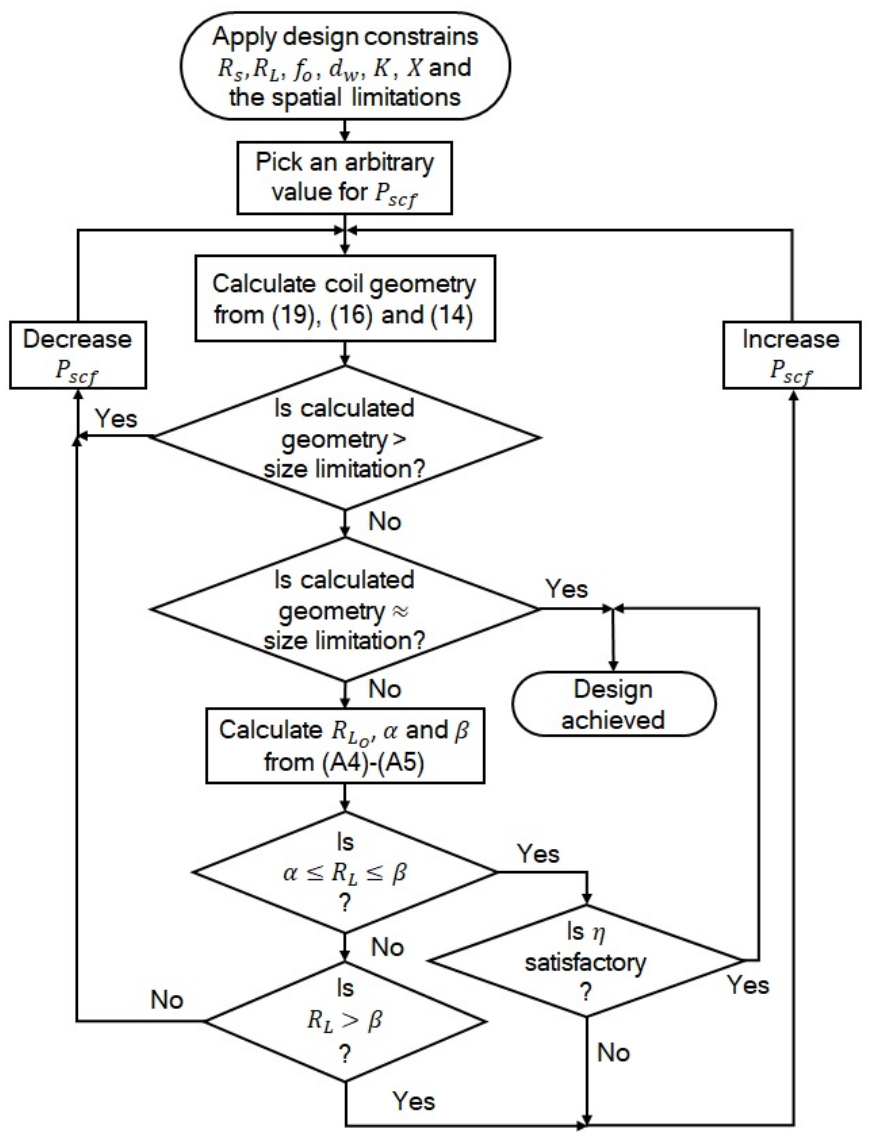

Fig. 6. Flowchart of design algorithm.

application's spatial limitations is $P_{\text {scf }}=150$. For this FoM value, the designed coil geometry is $r_{c}=5 \mathrm{~mm}, N=12$, and $l_{c}=10 \mathrm{~mm}$ and the maximum PTE of $85.70 \%$ is theoretically achieved.

\section{EXPERIMENTAL Results AND VALIDATION}

To validate the design procedure, described in Fig. 6, an arbitrary chosen IPT system with $R_{s}=3 \Omega, R_{L}=20 \Omega$ at $f_{o}=0.1 \mathrm{MHz}$ has been considered, where the $\mathrm{Tx} / \mathrm{Rx}$ coil diameter must be less than $92 \mathrm{~mm}$ (i.e., $r_{c} \leq 46 \mathrm{~mm}$ ). The Tx/Rx coupling coefficient, $K$, is 0.05 and the chosen proximity range of $\eta_{\max }$ is $3 \%$ (i.e., $X=3 \%$ ). The coil winding conductor used is $0.8-\mathrm{mm}$ (dia) copper wire. This typical
IPT system was chosen to facilitate practical, bench-top, investigation. The supplementary experimental results for a design example at higher frequency range has been mentioned in the abridged version of the present work [32], validating the proposed coil geometry optimization methodology over a wide radio frequency band.

Some iteration examples to find the optimal FoM value for the considered IPT system are listed in Table IV. Following the design procedure (see Fig. 6), the optimal FoM value for the given IPT system is $P_{\text {scf }}=3200$ (i.e., iteration 4 in Table IV). Selecting this value results in designing a coil pair with $r_{c}=45.70 \mathrm{~mm}, N=114$ and $l_{c}=91.40 \mathrm{~mm}$ which can provide maximum PTE of $84.11 \%$. Further specifications of the designed geometry including Tx/Rx coil's self-inductance, ohmic resistance, and $Q_{i}$-factors are mentioned in Table V. To practically investigate the achievable PTE of the coil geometry designed, a Tx/Rx coil pair for $P_{\text {scf }}=3200$ has been fabricated. The measured $L, R$, and $Q_{i}$ values of the prototyped solenoid pair are listed in Table V. The measurements are taken using an $L C R$ meter (R\&S HM8118). The deviations between calculated and measured values in Table $\mathrm{V}$ are attributed to the $L C R$ meter's basic accuracy and measurement errors. In addition to this, small mismatches are expected given the approximate equations utilized to model the helical coil's $L$ and $R$ [2], [39]-[41]. As presented in many geometry optimization techniques, an extremely high order of accuracy is not overly crucial, [1], [9], [20], [43]. Hence, for this work (i.e., high power, non-mm size helical coils), in modeling the coil's ac ohmic resistance the proximity effect losses are neglected to provide a workable design method. This is because, the proximity effect is caused due to the current crowding induced by the adjacent turns in a single layer coil (i.e., solenoid) [14]. In high frequency IPT systems, the influence of proximity effect on the resistance of a single turn of the solenoid is increased. However, with an increase in the IPT system's operating frequency the physical size of the coil becomes smaller (i.e., less number of turns) [20]. The cumulative proximity effect losses in these coils can be neglected where the solenoid is not in mm-size range and is used in high power (i.e., $\geq 1 \mathrm{~W}$ ) applications. In lower frequency range the proximity effect losses are negligible due to the IPT system's $f_{o}$.

The PTE measurements have been carried out on a seriesseries IPT system, as modeled in Fig. 1, where resonance 
TABLE IV

ALgorithm Iterations For IPT System Under Consideration $\left(f_{o}=0.1 \mathrm{MHz}, R_{s}=3 \Omega\right.$, AND $R_{L}=20 \Omega$ )

\begin{tabular}{ccccccccccc}
\hline \hline $\begin{array}{c}\text { Iteration } \\
\#\end{array}$ & $P_{\text {scf }}$ & $\begin{array}{c}R \\
(\Omega)\end{array}$ & $\begin{array}{c}r_{c} \\
(\mathrm{~mm})\end{array}$ & $N$ & $\begin{array}{c}l_{c} \\
(\mathrm{~mm})\end{array}$ & $\begin{array}{c}R_{L_{O}} \\
(\Omega)\end{array}$ & $\begin{array}{c}\alpha \\
(\Omega)\end{array}$ & $\begin{array}{c}\beta \\
(\Omega)\end{array}$ & $\begin{array}{c}\eta \text { for } \\
R_{L_{O}}(\%)\end{array}$ & $\begin{array}{c}\eta \text { for } \\
R_{L}(\%)\end{array}$ \\
\hline 1 & 500 & 0.55 & 32.70 & 81 & 65.20 & 3.80 & 2.25 & 6.41 & 74.7 & 54.07 \\
2 & 2500 & 0.98 & 43.70 & 109 & 87.40 & 11.40 & 6.02 & 21.61 & 84.11 & 82.16 \\
3 & 3500 & 1.11 & 46.50 & 116 & 93.00 & 14.39 & 7.38 & 28.09 & 85.62 & 85.00 \\
\hline 4 & 3200 & 1.08 & 45.70 & 114 & 91.40 & 13.37 & 6.93 & 25.79 & 85.07 & 84.11 \\
\hline \hline
\end{tabular}

TABLE V

Specifications of the Prototyped Geometry $\left(r_{c}=45.70 \mathrm{~mm}\right.$, $N=114, l_{c}=91.40 \mathrm{~mm}$ AT $\left.f_{o}=0.1 \mathrm{MHz}\right)$

\begin{tabular}{lcccccc}
\hline \hline & $\begin{array}{c}L_{p} \\
(\mathrm{mH})\end{array}$ & $\begin{array}{c}R_{c p} \\
(\Omega)\end{array}$ & $Q_{i p}$ & $\begin{array}{c}L_{s} \\
(\mathrm{mH})\end{array}$ & $\begin{array}{c}R_{c s} \\
(\Omega)\end{array}$ & $Q_{i s}$ \\
\hline Calculated value & 0.835 & 1.08 & 486.22 & 0.835 & 1.08 & 486.22 \\
Measured value & 0.794 & 1.80 & 280.18 & 0.802 & 1.85 & 270.1 \\
\hline \hline
\end{tabular}

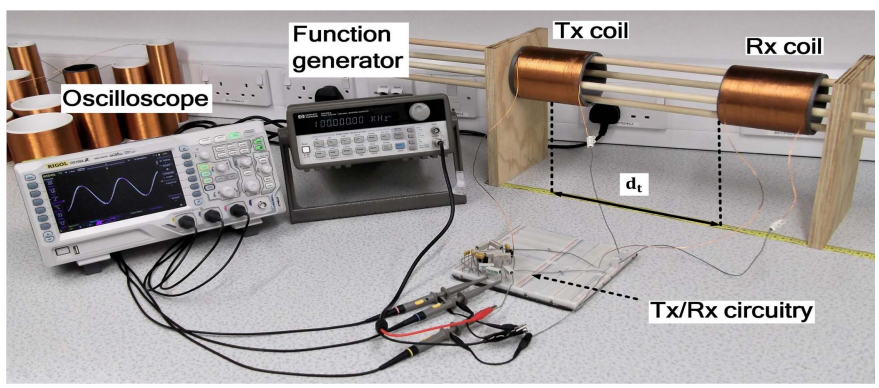

Fig. 7. Experimental PTE test setup (length of coil $=91.40 \mathrm{~mm}$, radius of coil $=45.70 \mathrm{~mm}$, and width apart, $d_{t}$, is varied)

capacitors, $R_{S}$ and $R_{L}$ are directly added to the primary and secondary coils. This technique permits sole PTE evaluation of the designed geometry [1], [43]. Fig. 7 shows the utilized experimental set up, where a function generator (HP 33120A) is used to drive the primary coil at the resonance frequency (i.e., $0.1 \mathrm{MHz}$ ). In order to probe the system's energy transmission efficiency, an oscilloscope (Rigol DS1054Z) is used to measure the voltage and current waveforms at the $\mathrm{Tx} / \mathrm{Rx}$ side [1], [43]. The transmitted power is simply found by multiplying the rms values of the measured voltage and current signals injected into the primary circuitry. The received power is also calculated by measuring the voltage across the load. For the designed geometry, the maximum achievable PTE is $85.07 \%$ at $R_{L_{O}}=13.37 \Omega$. Based on the physical circuit parameters developed in Table IV, for all load values between $\alpha$ and $\beta$ (i.e., $6.93 \Omega \leq R_{L} \leq 25.79 \Omega$ ) the system's PTE will be within $3 \%$ of $\eta_{\max }$ (i.e., $\geq 82.52 \%$ ). Fig. 8 shows the simulated PTE against increasing $R_{L}$ for the coil design; set beside this curve are the results taken from practical measurements as $R_{L}$ is physically increased from 3 to $33.1 \Omega$. The measured values show that for all the load resistances within the $\alpha$ and $\beta$ range, the system's PTE is higher than $80 \%$ (i.e., within $6 \%$ of $\eta_{\max }$ ). Although in modeling the coil's ac

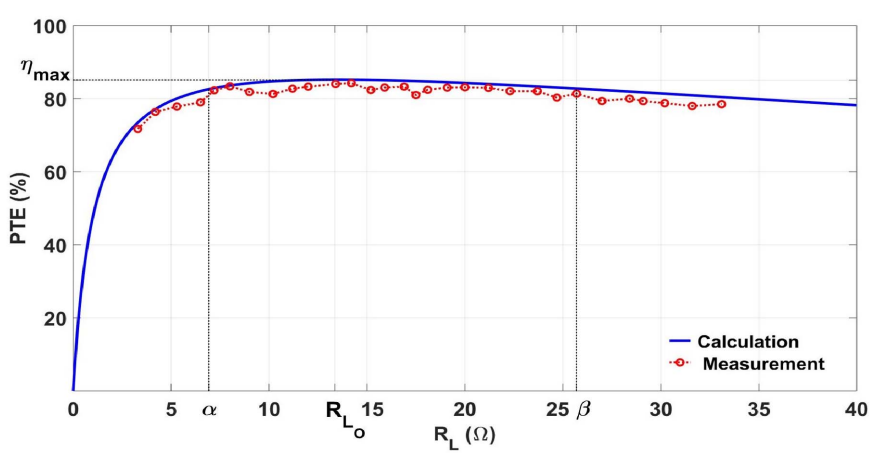

Fig. 8. Calculated and measured PTE variations over a nominal range of load values. The $\eta_{\max }, \alpha$, and $\beta$ values are indicated in Table IV (iteration 4).

ohmic resistance the proximity effect losses were neglected, the measured results from the physical system show correlation with the calculated results. The $3 \%$ mismatch between the calculated and measured PTE values are attributed to the fact that $\eta$, based on (3), is a function of both loaded and intrinsic quality factors (i.e., $Q_{L_{p}}, Q_{L_{s}}$, and $Q_{i}$ factors). The deviations in the value of $Q_{i}$ factor is compensated with intrinsic tolerance of $R_{S}$ and $R_{L}$ resistors (i.e., $Q_{L_{p}}$ and $Q_{L_{s}}$ factors). Also, the calculated and measured PTE for the IPT application (i.e., $R_{L}=20 \Omega$ ) are $84.11 \%$ and $83.10 \%$, respectively. The given IPT system delivers $1.06-\mathrm{W}$ power to the load when $V_{s}=3.53 v_{\text {rms }}$.

Although the Tx/Rx coil pair are designed for $K=0.05$, as described in Fig. 2, the designed geometry maximizes PTE over the range of $0 \leq K \leq 1$. To practically prove the concept, seven sets of coil, each with different $r_{c}$ values, have been fabricated and their respective PTEs measured for the IPT application. The four groups of different colored solid and dashed curves plotted in Fig. 9 correspond to calculated and measured PTEs of the designed geometry for four $K$ values between 0.0125 and 0.1 . The coupling coefficient range has been chosen again to facilitate practical, bench-top, investigation and ease of results gathering. As can be seen in Fig. 9, with a fall in coupling coefficient the link becomes more loosely-coupled but the maximum PTE becomes apparent as being around the designed coil $r_{c}$ value (i.e., $45.70 \mathrm{~mm}$ ). The experimental PTE value shows a marked closeness to the analytical calculations using MATLAB. Practical tests on physical geometries either side of $r_{c}=45.70 \mathrm{~mm}$ show falling PTE over different $K$ values, thus validating the design method proposed. 


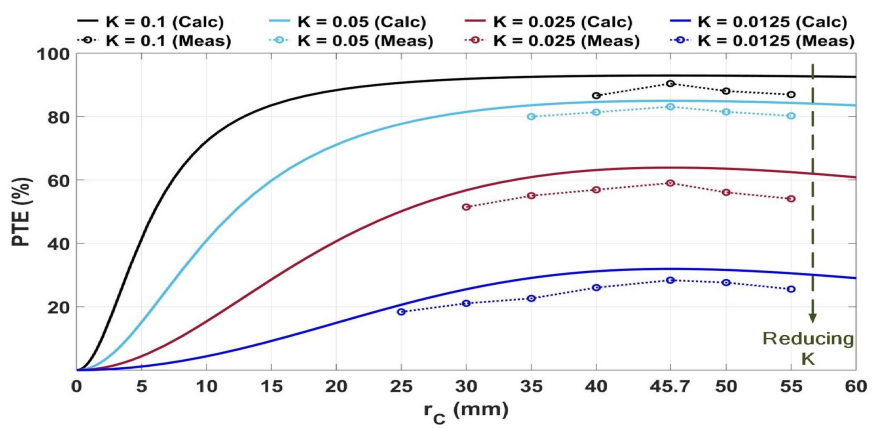

Fig. 9. Calculated and measured PTE over four $K$ values from 0.0125 to 0.1 .

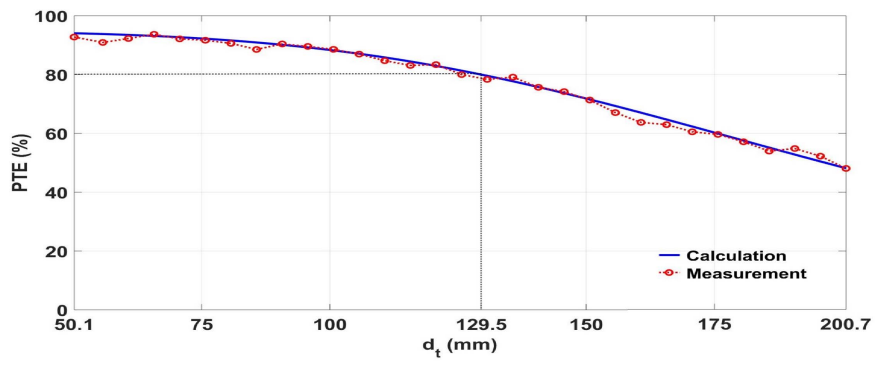

Fig. 10. Comparison of PTE between practical measurements and theoretical results for the designed coil pair.

Furthermore, it is worth mentioning that $K$ is highly dependent on the relative distance between the Tx/Rx coil pairs. To evaluate the functionality of the designed geometry with transmission distance, $d_{t}$, variations, calculated and measured PTE values over a $d_{t}$ range from 50.1 to $200.7 \mathrm{~mm}$ (i.e., $0.0176 \leq K \leq 0.1845$ ) have been compared in Fig. 10 . For the single-axis Tx/Rx coil pair, as demonstrated in Fig. 7, $d_{t}$ is the distance from Tx coil center to Rx coil edge [39]. From Fig. 10, it can be deduced that with an increase in transmission distance, the practically measured PTE values attenuate, as can be predicted from the theoretical equations. It is also observed that for separation distances nearly three times the coil's radius (i.e., $d_{t}=129.5 \mathrm{~mm}$ ) the PTE stays higher than $80 \%$, which agrees well with signal attenuation observations in near-field communications [2] and [32].

\section{CONCLUSION}

Geometrical optimization of Tx and Rx coil pairs is considered to maximize PTE in resonant IPT systems. A design parameter, known as strong coupling factor $\left(P_{\text {scf }}\right)$, is employed to assist in the design of optimized coils. In order to select the most favorable numerical value for $P_{\text {scf }}$ a recursive algorithm is introduced which maximizes PTE based on the IPT system application and physical size limitations. An IPT system at $0.1 \mathrm{MHz}$ was simulated using MATLAB and a PTE of $84.11 \%$ calculated. The theoretical method was validated by developing a prototype $\mathrm{Tx} / \mathrm{Rx}$ coil pair, which yielded a PTE of $83.10 \%$ at $K=0.05$. To increase the accuracy of the design equations proposed here, future work on this topic may include both skin-effect and proximity-effect losses.

\section{APPENDIX}

Finding the load range where $\eta$ of the IPT system stays within $X \%$ of the maximum achievable PTE requires solving the following inequality:

$$
\eta=(1-X) \eta_{\max }
$$

Maximum PTE (i.e., $\eta_{\max }$ ) can be achieved by substituting $R_{L}$ with $R_{L_{O}}$ in (6); hence, (A1) can be restated as

$$
\begin{aligned}
& R_{L}\left(R_{L_{o}}+R\right)\left[\left(R_{s}+R\right)\left(R_{L_{o}}+R\right)+\omega_{o}^{2} M^{2}\right]-(1-X) \\
& R_{L_{o}}\left(R_{L}+R\right)\left[\left(R_{s}+R\right)\left(R_{L}+R\right)+\omega_{o}^{2} M^{2}\right] \geq 0 .
\end{aligned}
$$

Rearranging (A2) based on $R_{L}$ gives

$$
P_{1} R_{L}^{2}+P_{2} R_{L}+P_{3} \geq 0
$$

where

$$
\begin{aligned}
P_{1}= & -(1-X)\left(R+R_{s}\right) R_{L_{o}} \\
P_{2}= & \left(R+R_{s}\right) R_{L_{O}}^{2}+\left(R+R_{S}\right) R^{2}+2 R R_{L_{o}} \\
& +(X+1) \omega_{o}^{2} M^{2} R R_{L_{O}}+2(X-1) R_{L_{O}} R^{2} \\
& +2(X-1) R R_{S} R_{L_{O}} \\
P_{3}= & (X-1)\left(R+R_{S}\right) R_{L_{O}} R^{2}+(X-1) \omega_{o}^{2} M^{2} R R_{L_{o}} .
\end{aligned}
$$

Solving (A3) for $R_{L}$ yields

$$
\alpha \leq R_{L} \leq \beta
$$

where

$$
\begin{aligned}
& \alpha=\frac{-P_{2}+\sqrt{P_{2}^{2}-4 P_{1} P_{3}}}{2 P_{1}} \\
& \beta=\frac{-P_{2}-\sqrt{P_{2}^{2}-4 P_{1} P_{3}}}{2 P_{1}} .
\end{aligned}
$$

\section{ACKNOWLEDGMENT}

The authors would like to thank Dr. R. Prabhu for his advice and D. Smith for his technical assistance. They would also like to thank the School of Engineering, Robert Gordon University, Aberdeen, U.K., for the support of this research, and also the editorial board members and reviewers for their timely and thoughtful comments.

\section{REFERENCES}

[1] U.-M. Jow and M. Ghovanloo, "Design and optimization of printed spiral coils for efficient transcutaneous inductive power transmission," IEEE Trans. Biomed. Circuits Syst., vol. 1, no. 3, pp. 193-202, Sep. 2007.

[2] A. Kurs, A. Karalis, R. Moffatt, J. D. Joannopoulos, P. Fisher, and M. Soljačić, "Wireless power transfer via strongly coupled magnetic resonances," Sci. Express, vol. 317, pp. 83-86, Jul. 2007.

[3] A. Basir and H. Yoo, "Efficient wireless power transfer system with a miniaturized quad-band implantable antenna for deep-body multitasking implants," IEEE Trans. Microw. Theory Techn., vol. 68, no. 5, pp. 1943-1953, May 2020.

[4] R. Shadid, M. Haerinia, S. Roy, and S. Noghanian, "Hybrid inductive power transfer and wireless antenna system for biomedical implanted devices," Prog. Electromagn. Res. C, vol. 88, pp. 77-88, Nov. 2018.

[5] A. Ibrahim and M. Kiani, "A figure-of-merit for design and optimization of inductive power transmission links for millimeter-sized biomedical implants," IEEE Trans. Biomed. Circuits Syst., vol. 10, no. 6, pp. 1100-1111, Dec. 2016.

[6] D. Ahn and M. Ghovanloo, "Optimal design of wireless power transmission links for millimeter-sized biomedical implants," IEEE Trans. Biomed. Circuits Syst., vol. 10, no. 1, pp. 125-137, Feb. 2016.

[7] P. Feng, P. Yeon, Y. Cheng, M. Ghovanloo, and T. G. Constandinou, "Chip-scale coils for millimeter-sized bio-implants," IEEE Trans. Biomed. Circuits Syst., vol. 12, no. 5, pp. 1088-1099, Oct. 2018. 
[8] J. Kim and Y. Rahmat-Samii, "Implanted antennas inside a human body: Simulations, designs, and characterizations," IEEE Trans. Microw. Theory Techn., vol. 52, no. 8, pp. 1934-1943, Aug. 2004.

[9] J. Sallan, J. L. Villa, A. Llombart, and J. F. Sanz, "Optimal design of ICPT systems applied to electric vehicle battery charge," IEEE Trans. Ind. Electron., vol. 56, no. 6, pp. 2140-2149, Jun. 2009.

[10] C.-S. Wang, O. H. Stielau, and G. A. Covic, "Design considerations for a contactless electric vehicle battery charger," IEEE Trans. Ind. Electron., vol. 52, no. 5, pp. 1308-1314, Oct. 2005.

[11] J. Kim et al., "Coil design and shielding methods for a magnetic resonant wireless power transfer system," Proc. IEEE, vol. 101, no. 6, pp. 1332-1342, Jun. 2013.

[12] N. S. Jeong and F. Carobolante, "Wireless charging of a metalbody device," IEEE Trans. Microw. Theory Techn., vol. 65, no. 4, pp. 1077-1086, Apr. 2017.

[13] S. Y. Hui, "Planar wireless charging technology for portable electronic products and Qi," Proc. IEEE, vol. 101, no. 6, pp. 1290-1301, Jun. 2013.

[14] Y. Cheng, G. Wang, and M. Ghovanloo, "Analytical modeling and optimization of small solenoid coils for millimeter-sized biomedical implants," IEEE Trans. Microw. Theory Techn., vol. 65, no. 3, pp. 1024-1035, Mar. 2017.

[15] Y. J. Hwang and J. Y. Jang, "Design and analysis of a novel magnetic coupler of an in-wheel wireless power transfer system for electric vehicles," Energies, vol. 13, no. 2, p. 332, Jan. 2020.

[16] A. Robichaud, M. Boudreault, and D. Deslandes, "Comparison between inductance topologies for resonant wireless power transmission applications," in Proc. Asia-Pacific Microw. Conf., Dec. 2012, pp. 397-399, doi: 10.1109/APMC.2012.6421610.

[17] Y. Cheng, G. Chen, D. Xuan, G. Qian, M. Ghovanloo, and G. Wang, "Analytical modeling of small, solenoidal, and implantable coils with ferrite tube core," IEEE Microw. Wireless Compon. Lett., vol. 29, no. 3, pp. 237-239, Mar. 2019.

[18] A. Ahmad, M. S. Alam, and A. A. S. Mohamed, "Design and interoperability analysis of quadruple pad structure for electric vehicle wireless charging application," IEEE Trans. Transport. Electrific., vol. 5, no. 4, pp. 934-945, Dec. 2019.

[19] S. Bhattacharya and Y. K. Tan, "Design of static wireless charging coils for integration into electric vehicle," in Proc. IEEE 3rd Int. Conf. Sustain. Energy Technol. (ICSET), Sep. 2012, pp. 146-151, doi: 10.1109/ICSET.2012.6357389.

[20] S. V. de Campos de Freitas, F. C. Domingos, R. Mirzavand, A. Maunder, P. Naseri, and P. Mousavi, "A novel method for data and power transmission through metallic structures," IEEE Trans. Ind. Electron., vol. 64, no. 5, pp. 4027-4036, May 2017.

[21] M. Heidarian, S. J. Burgess, and R. Prabhu, "Improving the design approach to developing through-metal communication for use in subsea pipeline robots," in Proc. Global OCEANS, Singapore-U.S. Gulf Coast, 2020.

[22] T. A. Vu, C. V. Pham, A. V. Pham, and C. S. Gardner, "Wireless power transfer through metal using inductive link," Int. J. Power Electron. Drive Syst., vol. 10, no. 4, pp. 1906-1913, Dec. 2019.

[23] A. Pacini, F. Mastri, R. Trevisan, D. Masotti, and A. Costanzo, "Geometry optimization of sliding inductive links for position-independent wireless power transfer," in IEEE MTT-S Int. Microw. Symp. Dig., May 2016, pp. 1-4, doi: 10.1109/MWSYM.2016.7540073.

[24] X. Huang, K. D. T. Ngo, and G. Bloom, "Design techniques for planar windings with low resistance," in Proc. 10th Annu. Appl. Power Electron. Conf. Expo., 1995, pp. 533-539, doi: 10.1109/APEC.1995.469073.

[25] J. Craninckx and M. S. J. Steyaert, "A 1.8-GHz low-phase-noise CMOS VCO using optimized hollow spiral inductors," IEEE J. Solid-State Circuits, vol. 32, no. 5, pp. 736-744, May 1997.

[26] S. R. Cove, M. Ordonez, N. Shafiei, and J. Zhu, "Improving wireless power transfer efficiency using hollow windings with track-width-ratio," IEEE Trans. Power Electron., vol. 31, no. 9, pp. 6524-6533, Sep. 2016.

[27] Y. Su, X. Liu, C. K. Lee, and S. Y. Hui, "On the relationship of quality factor and hollow winding structure of coreless printed spiral winding (CPSW) inductor," IEEE Trans. Power Electron., vol. 27, no. 6, pp. 3050-3056, Jun. 2012.

[28] F. Jolani, Y. Yu, and Z. Chen, "A planar magnetically coupled resonant wireless power transfer system using printed spiral coils," IEEE Antennas Wireless Propag. Lett., vol. 13, pp. 1648-1651, 2014.

[29] W. H. Ko, S. P. Liang, and C. D. F. Fung, "Design of radio-frequency powered coils for implant instruments," Med. Biol. Eng. Comput., vol. 15, no. 6, pp. 634-640, Nov. 1977.
[30] N. D. N. Donaldson and T. A. Perkins, "Analysis of resonant coupled coils in the design of radio frequency transcutaneous links," Med. Biol. Eng. Comput., vol. 21, no. 5, pp. 612-627, Sep. 1983.

[31] M. Heidarian, S. J. Burgess, R. Prabhu, and N. Fough, "Optimal coil design for maximum power transfer efficiency in resonantly coupled systems," in Proc. USNC-URSI Radio Sci. Meeting (Joint With AP-S Symp.), Jul. 2019, pp. 73-74, doi: 10.1109/USNC-URSI.2019.8861834.

[32] M. Heidarian, S. J. Burgess, R. Prabhu, and N. Fough, "Maximising inductive power transmission using a novel analytical coil dsign approach," in Proc. IEEE Wireless Power Transf. Conf. (WPTC), Jun. 2019, pp. 158-161, doi: 10.1109/WPTC45513.2019.9055553.

[33] I. Ghotbi, M. Najjarzadegan, S. J. Ashtiani, O. Shoaei, and M. Shahabadi, "3-coil orientation insensitive wireless power transfer for capsule endoscope," in Proc. 23rd Iranian Conf. Electr. Eng., May 2015, pp. $1249-1254$.

[34] W. X. Zhong and S. Y. R. Hui, "Maximum energy efficiency tracking for wireless power transfer systems," IEEE Trans. Power Electron., vol. 30, no. 7, pp. 4025-4034, Jul. 2015.

[35] N. O. Sokal and A. D. Sokal, "Class E-A new class of high-efficiency tuned single-ended switching power amplifiers," IEEE J. Solid-State Circuits, vol. SSC-10, no. 3, pp. 168-176, Jun. 1975.

[36] $\mathrm{P}$. Wu et al., "High-efficient rectifier with extended input power range based on self-tuning impedance matching," IEEE Microw. Wireless Compon. Lett., vol. 28, no. 12, pp. 1116-1118, Dec. 2018.

[37] Y. Huang, N. Shinohara, and T. Mitani, "Impedance matching in wireless power transfer," IEEE Trans. Microw. Theory Techn., vol. 65, no. 2, pp. 582-590, Feb. 2017.

[38] W. Zhong and S. Y. Hui, "Reconfigurable wireless power transfer systems with high energy efficiency over wide load range," IEEE Trans. Power Electron., vol. 33, no. 7, pp. 6379-6390, Jul. 2018.

[39] M. N. Sadiku, Elements of Electromagnetics, 7th ed. London, U.K.: Oxford Univ. Press, 2018.

[40] C. A. Balanis, Antenna Theory: Analysis and Design, 4th ed. Hoboken, NJ, USA: Wiley, 2016.

[41] Y. Lee, "RFID coil design," Microchip Technol., Chandler, AZ, USA, Appl. Note AN678, 1998.

[42] K. Finkenzeller, RFID Handbook. Hoboken, NJ, USA: Wiley, 2010, pp. 66-70.

[43] M. Kiani, U.-M. Jow, and M. Ghovanloo, "Design and optimization of a 3-coil inductive link for efficient wireless power transmission," IEEE Trans. Biomed. Circuits Syst., vol. 5, no. 6, pp. 579-591, Dec. 2011.

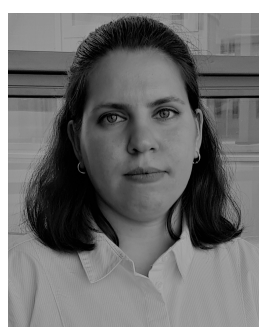

Maryam Heidarian (Graduate Student Member, IEEE) was born in Iran. She received the B.Eng. degree (Hons.) in electrical engineering from Islamic Azad University (South Tehran Branch), Tehran, Iran, in 2007, and the M.S. degree (Hons.) in electrical engineering from the National University of Sciences and Technology (NUST), Islamabad, Pakistan, in 2013. She is currently pursuing the $\mathrm{Ph} . \mathrm{D}$. degree in electrical engineering at Robert Gordon University (RGU), Aberdeen, U.K.

She has over five years of industrial experience in the area of control systems and telecommunication. Her current research interests include wireless power transfer and signal communication.

Ms. Heidarian has received numerous awards and scholarships, including the President Gold Medal from NUST in 2013 and the Three-Year FullStudentship from RGU.

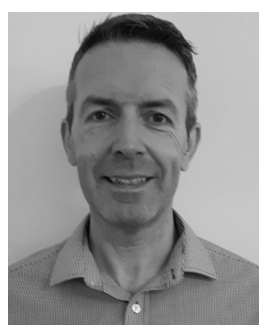

Samuel J. Burgess was born in U.K., in 1969. He received the B.Eng. degree (Hons.) in electronic systems and the D.Phil. degree in radio frequency studies from Ulster University, Jordanstown, U.K., in 1996 and 1999, respectively.

From 2000 to 2003, he worked with the Radio Frequency Group, Parthus Technologies, Dublin, Ireland, as a Project Engineer on the design of submicron CMOS circuits for wireless applications. From late 2003 to 2014, he was a Lecturer with Ulster University in the area of electronics and communications. Since late 2014, he has been with Robert Gordon University, Aberdeen, U.K., where he is currently an Academic Strategic Lead and the Head of electronic and electrical discipline with the School of Engineering. He has 17 years of university experience and nine years of industrial experience. His teaching and research interests include radio frequency design, wireless control systems, analog electronics, and sensor systems. 